\title{
A New Technique Based on Intensity Distribution Graphs for the Extraction of Traffic Flow Rate and Vehicle Speed
}

\author{
G. Abo Samra and O. AbdulKader \\ Faculty of Computing and Information Technology, \\ King Abdulaziz University, Jeddah, Saudi Arabia. \\ gabosamra@kau.edu.sa
}

\begin{abstract}
This paper introduces a new visual system which allows analysis of traffic behavior on freeways and highways, using a video camera mounted on a relatively high place (such as a bridge). A new technique based on spatial and temporal intensity distribution is introduced to determine the road traffic parameters: average speed (AVSP) and average flow rate (AVFL). After performing many steps including automatic detection of the Region Of Interest (ROI) and camera orientation, background subtraction and Inverse Perspective Mapping (IPM), an Intensity Distribution Graph (IDG) is built for the current frame. The (IDG) is then transformed into a single Grayscale Image Line (GIL). A certain number $(\mathrm{N})$ of GILs representing $\mathrm{N}$ frames are concatenated to build a Vehicle Tracking Diagram image (VTD) which gives a whole view of the traffic flow in the ROI during a specific period $T$. Using the VTD we can visualize the vehicles trajectories, speeds and estimate AVFL. By applying edge detection and Hough Line transform on the VTD, AVSP is estimated. The system has been implemented using MATLAB as an offline application. The results were encouraging, reaching $97 \%$ accuracy for the AVSP and AVFL. Further code optimization is needed for online monitoring.
\end{abstract}

Keywords: Traffic parameters; traffic flow; vehicle speed; inverse perspective mapping; machine vision; traffic monitoring, traffic surveillance.

\section{1- Introduction}

In recent years, vast research has been done in traffic parameters extraction applications to automate road traffic data collection and 
analysis and to control traffic signals ${ }^{[1,2]}$. Approaches to traffic monitoring systems can be classified into one of two major categories: Spot monitoring and area monitoring systems. Microwave transducer, radar, infrared detectors and underground magnetic loop sensors are examples of spot monitoring systems. Area monitoring systems generally rely on video cameras. However, most of the signals acquired from spot sensors have to be interpreted. By contrast, images from surveillance cameras can be viewed directly by operators, so no interpretation is needed for image sensors. A major problem associated with a real-time vision-based application such as traffic data collection is the requirement of high computational power ${ }^{[3]}$. In traffic applications, variation of ambient lighting, shadows, occlusion, and lane changes of moving vehicles complicates the task. Video systems for traffic monitoring normally involve two major tasks of perception: (a) Estimation of road geometry and (b) vehicle and obstacle detection. Thus, for traffic monitoring it is essential to detect the edges of the road and then sense and identify presence and/or motion parameters of a vehicle ${ }^{[3]}$. In traffic monitoring video systems, a static camera observes a dynamic road scene for the purpose of traffic surveillance. In this case, the static camera generally has a good view of the road objects because of the high position of the camera. Therefore, 2D intensity images may contain enough information for the model-based recognition of road objects ${ }^{[4]}$. Hence, the road and the objects (vehicles) have to be detected on the image plane, at the camera coordinates.

A brief survey of main vision based approaches will be given in Section 2. The proposed approach will be illustrated in detail in Section 3. Results will be presented in Section 4. Finally the conclusion will be given in Section 5.

\section{A survey of main vision based approaches}

Vision based approaches can be classified into three main categories: object tracking approach, optical flow approach and statistical approach. Brief description of each approach will be given in Sections 2.1 to 2.3 .

\subsection{Object Tracking Approach}

The objective of tracking is to obtain the positions of a specific object in the successive frames. The tracking starts by the calculation of 
object displacement between two consecutive frames. Continuation of finding correspondence for the next two consecutive frames, while using the previous result as a new object, makes up the tracking process. Most of object detection methods involve background subtraction techniques to detect and track the objects ${ }^{[4]}$. Object tracking can be feature based or window based as will be illustrated in the following subsections.

\subsubsection{Feature-Based Object Tracking}

Feature-based tracking systems depend on using vehicle characteristics like color, structure, dimensions...etc to track the designated vehicle. Coifman et al. developed a feature-based tracking system for detecting and tracking vehicles ${ }^{[5]}$. The drawback of Coifman et al.'s approach is that a camera calibration process is required.

Erhan $\mathrm{INCE}^{[6]}$ proposed a vehicle tracking system that uses moments to detect vehicles. No real data were used for evaluating his technique and a high computational requirement was recorded (about $10 \mathrm{~s}$ per frame) because of using complex background detection techniques.

\subsubsection{Window-Based Object Tracking}

Fathy and Siyal ${ }^{[3]}$ introduced a vehicle-detection technique based on edge detection and key region processing. Fathy and Siyal used the extracted information for measuring other traffic parameters such traffic volume, types of vehicles, queue parameters and movements of vehicles. Because of their approach, their method requires pre-placing of two detection windows in a key region that extends across the lane. In addition, the distance between the two regions being monitored must be measured in advance.

\subsection{Optical Flow Approach}

Optical flow is the distribution of apparent velocities of movement of brightness patterns in an image. Optical flow can arise from relative motion of objects and the viewer. Consequently, optical flow can give important information about the spatial arrangement of the objects viewed and the rate of change of this arrangement ${ }^{[7]}$.

In [8], an improved method for long-term vehicle tracking from aerial video images is presented. A tracking method has been developed based on the optical flow method incorporating a scale space approach. 
The scale space approach delivers initial values for finding correspondences in the next frame. A decision step is also employed to continue the tracking or stop it because of low quality. In such case the tracking is continued by detecting the missed vehicle in the next frame. The major drawback of this method is time-consuming.

\subsection{Statistical Approach}

In statistical approaches, a global statistical quantity can be used as a monitoring parameter for the amount of objects in the image such as the entropy of the 2D image. Also, the pixel intensity mean and variance can be an indicative parameter of object movement in camera based approaches. Following are two examples of statistical approaches used to extract some traffic parameters.

\subsubsection{Virtual Inductive Loop Approach}

Viarani ${ }^{[9]}$ presented an approach, called 'virtual inductive loop', to extract traffic parameters. When a vehicle passes through a preset detection window, it produces a variation of the pixel grey tone. Then, the differences between reference windows of an updated background and the actual windows are computed. When the sum of the abovementioned differences is greater than a threshold, the counter is triggered for vehicle counting. However, Viarani's approach is vulnerable to different lighting conditions. This evaluation can be verified by the results recorded in [10] which gave a range of accuracy from $70.3 \%$ to $90.4 \%$.

\subsubsection{Entropy Based Approach}

Entropy is a macro-parameter that is commonly adopted to describe the degree of disorder of a system. In thermodynamics, the entropy of a system is usually defined as follows:

$$
\mathrm{S}=\mathrm{k} \ln (\Omega)
$$

where $\Omega$ is the probability of thermodynamics. $\mathrm{k}$ and $\mathrm{S}$, respectively, represent a constant and the entropy. The value of entropy is an indicator showing the degree of disorder. When the internal energy of a system is increased, its corresponding entropy is increased as well. Usually, stability in a system will be reached when the constituent molecules within the system are uniformly distributed. Under these circumstances, the value of entropy reaches its maximum. Shannon's entropy, which is commonly used in signal/image processing, is based on the concept that 
the gain in information from an event is inversely proportional to the probability of occurrence.

Jeng and $F^{[11]}$ described a real-time vision-based traffic monitoring system and also applied a macroscopic detection method to detect entropy of every image frame to compute traffic flow, space mean speed and determine the length of a traffic queue.

\section{The Proposed System Overview}

Our approach is based on intensity distribution after background subtraction for each frame which is used to synthesize different kinds of graphs for a specified period. The proposed traffic parameters extraction system consists of three main phases. These phases are shown in the following System Block Diagram in Fig. 1.

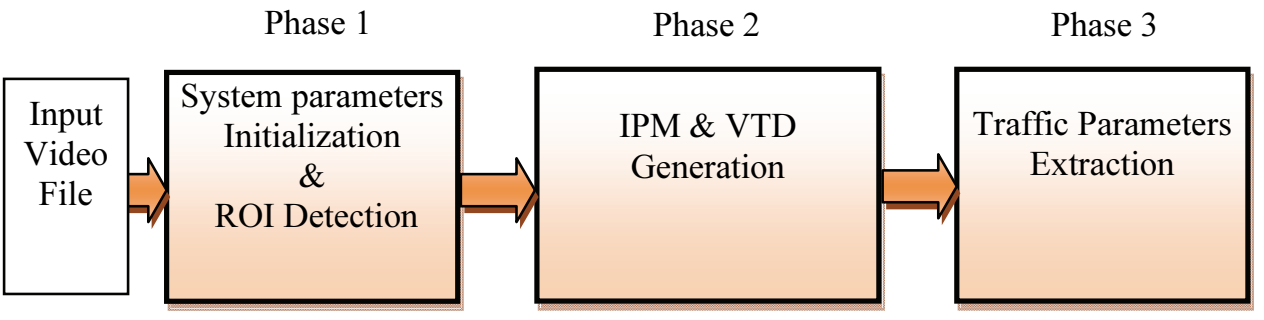

Fig. 1. System Block Diagram.

In the following subsections each phase of the proposed system will be described in detail.

\subsection{Phase 1: System Parameter Initialization and Region of Interest Detection}

In our system, an initialization phase is required to construct an initial background for the system, estimate important parameters required for the system, and to detect the Region Of Interest (ROI) on which all processing will be performed. This phase is accomplished using some image processing techniques with some constraints to separate the region of interest from the entire image.

\subsubsection{Automatic Detection of the Region of Interest}

To detect the region of interest we adopt the approach presented in [12] where edge-detection is performed followed by Hough transform step to detect the longest lines of the image. A selection step is then 
performed based on geometrical constraints to detect the road edges. The output of this step is shown in Fig. 2.

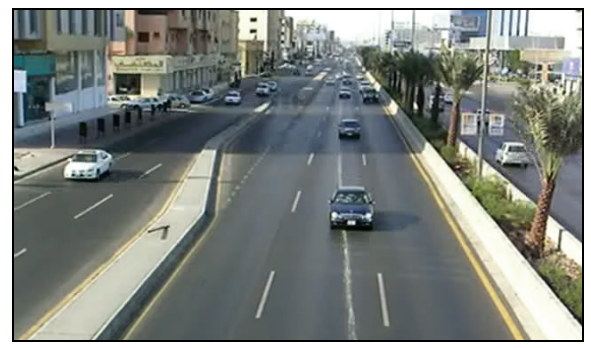

(a): The Input Full Image.

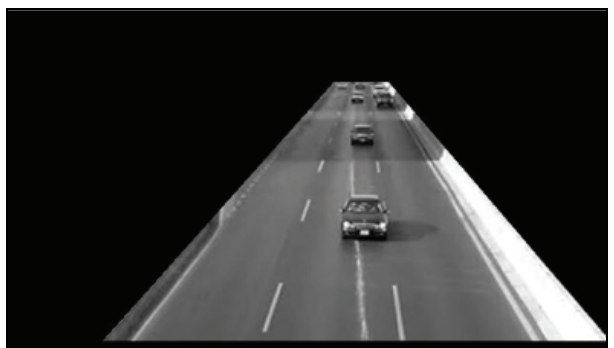

(b): The Detected ROI in (a).

Fig.2. The process of detecting the ROI.

\subsubsection{Detecting and Updating the Background}

There are many techniques to detect and update the background image, many of them use complex procedures to get an accurate background and to remove lighting and shadow effects. In our approach, we adopted the technique used in [13]. The basic idea in background adaptation is to integrate the new incoming information into the current background image. This is done by using the following average filter formalism:

$$
\mathrm{BG}_{\mathrm{t}}=0.8 * \mathrm{BG}_{\mathrm{t}-1}+0.2 * \operatorname{Img}_{\mathrm{t}}
$$

where $\mathrm{BG}_{\mathrm{t}}$ denotes the current background image and $\mathrm{BG}_{\mathrm{t}-1}$ is the pervious background and $\operatorname{Img}_{t}$ is current image frame.

\subsubsection{System Parameters Estimation}

To get the external parameters concerning the camera orientation, we estimate the coordinate of the vanishing point $X V, Y V$ from the intersection of the lines representing the road edges. Then, from ${ }^{[14]}$, we estimate the camera pitch angle $(\theta)$ and yaw angle $(\gamma)$ as follows:

$$
\begin{gathered}
\theta=\tan ^{-1}\left(\left(\frac{1-2 * Y V}{H g}\right) * \propto_{v}\right. \\
\gamma=\tan ^{-1}\left(\left(\frac{2 * X V}{W d-1}\right) * \propto_{h}\right.
\end{gathered}
$$

where $\mathrm{Hg}$ and $\mathrm{Wd}$ are the height and width of the camera image in pixels respectively and $\propto_{v}$ and $\propto_{h}$ are internal parameters specifying the used camera's aperture vertical angle and horizontal angle. 
Figure 3 depicts the relation between internal and external parameters of the camera with respect to the road orientation.

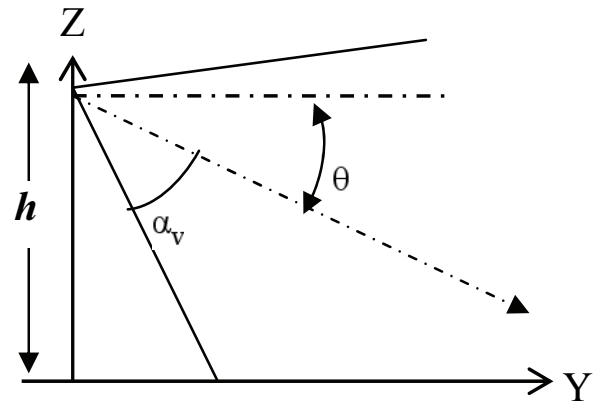

(a)

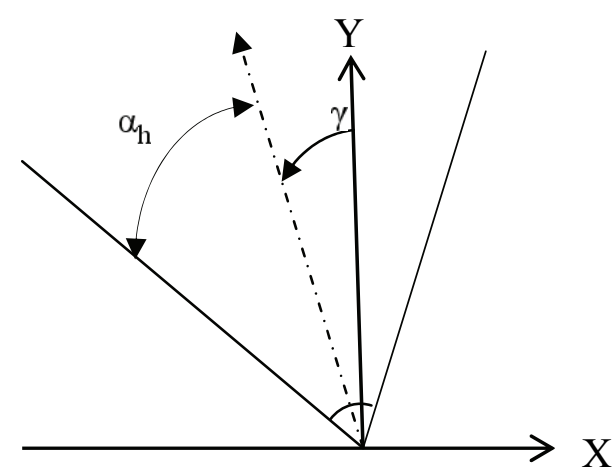

(b)

Fig. 3. The relation between road plane and rotation angles: (a) Pitch angle $\theta$; (side view) and (b) Yaw angle $\gamma$ (top view).

The period of the first ten frames of the system is an initialization period where the background image is updated gradually to get an initial background image. Another step is performed after background subtraction and IPM to extract the vehicles from the frames using an 8connected component analysis technique. The goal of this step is to estimate average vehicle width and length in pixels (AVWP and AVLP). The average actual length of the vehicle in meters (ACLM) is assumed 4.25. The ratio of the ACLM over AVLP is used as a scale factor to map image dimensions in pixels into world dimensions in meters.

\subsection{Phase 2: Inverse Perspective Mapping \& Vehicle Tracking Diagram Generation}

The second phase is the main phase, which is considered as the core of the system. This phase goes through different processing steps. At first, we separate the moving objects from the background using background subtraction, then an Inverse Perspective Mapping (IPM) is used to construct a new image from which we can get actual (or proportional to actual) traffic parameters. The last step in this phase is done gradually to build what we called the Vehicle Tracking Diagram (VTD), from which we can extract different traffic parameters. 


\subsubsection{Objects Separation}

Objects are separated simply by taking the difference between the current frame image and the background image. The output of this process is a black and white image which is subjected to some morphological operations such as dilation and erosion to remove noise and fill gaps inside objects. A sample output is shown in Fig. 4.

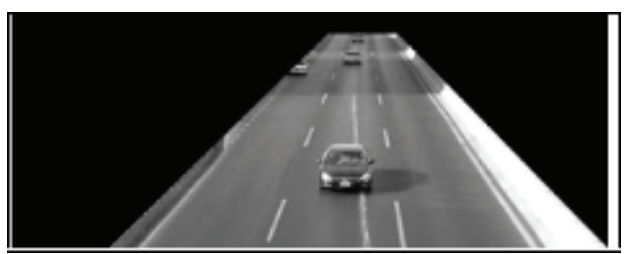

a: Current image frame.

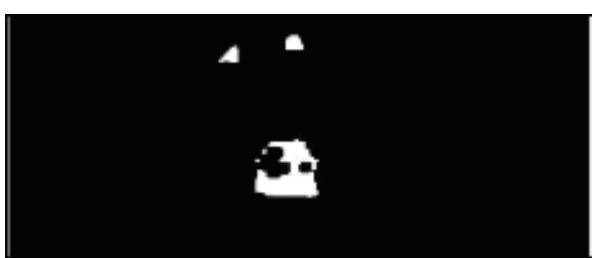

b: image after background subtraction.

Fig. 4. Vehicle separation using background subtraction.

\subsubsection{Inverse Perspective Mapping (IPM)}

IPM is a geometrical transformation technique that projects each pixel of the 2D perspective view of a $3 \mathrm{D}$ object, and re-maps it to a new position and constructs a new image on an inverse $2 \mathrm{D}$ planar ${ }^{[15]}$. Figure 5 depicts the side view of the geometrical model which shows differences between the dimensions of the object in the real plane $\left(\mathrm{L}_{\mathrm{r}}\right)$ and that at the image plane $\left(\mathrm{L}_{\mathrm{i}}\right)$, IPM is involved to remap the object and return it to its real dimensions.

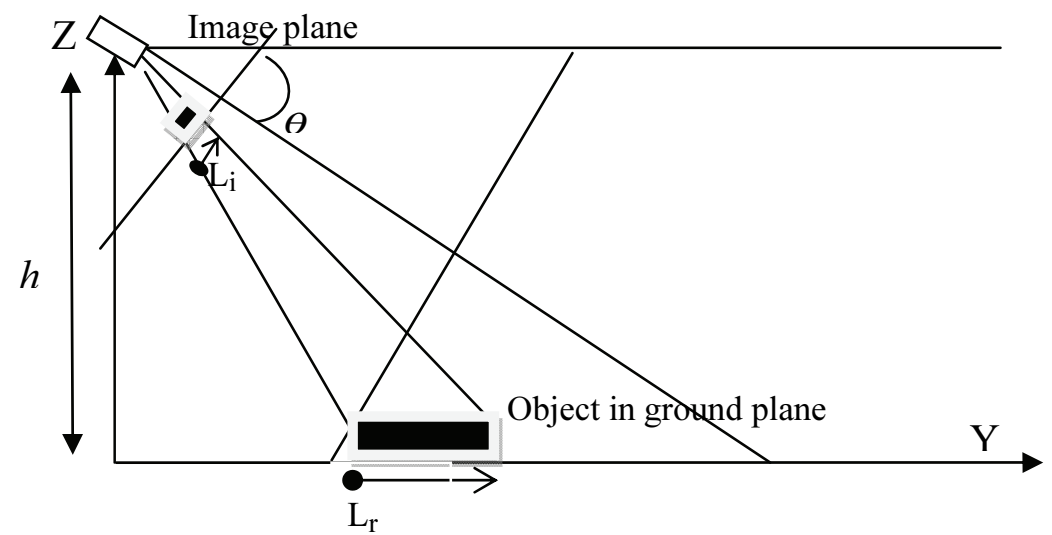

Fig. 5. Side view of the geometrical model. 
To perform IPM, we have adopted the technique described in [16]; where the road is assumed flat, the camera optical axis is assumed to be parallel to the road edges and the yaw angle is assumed to be zero. This will map the rectangular shape of the road into a trapezoid in image coordinates, as illustrated in Fig. 6.

Hence, as stated in [16], the problem resumes to plane-to-plane mapping, achieved by the following Equation:

$$
Y=\frac{v}{a-g v} ; X=-\frac{d v-a u}{e(a-g v)}
$$

Where $(X, Y)$ are the coordinates of one point in the first plane (in our case, on the road), and $(u, v)$ are the corresponding coordinates in the second plane (the image).

The unknown parameters in equation (5) are given by:

$$
\begin{aligned}
& a=\frac{u_{0}+u_{1} L}{u_{2}+u_{3} R}, d=\frac{u_{0} u_{3}-u_{1} u_{2}}{\left(u_{2}+u_{3}\right) R} \\
& e=\frac{u_{0}+u_{1}}{W} \quad, g=\frac{u_{0}+u_{1}-\left(u_{2}+u_{3}\right)}{\left(u_{2}+u_{3}\right) R}
\end{aligned}
$$

Image points $\left(-u_{0}, v_{0}\right),\left(u_{1}, v_{1}\right),\left(-u_{2}, v_{2}\right)$ and $\left(u_{3}, v_{3}\right)$ can be obtained directly using the linear portion of the lane boundary model, and $W$ is the expected road width (which must be informed). Parameter $L$ can be trivially computed from image points and an automatic procedure is applied as in [16] to obtain the remaining parameter $R$ (the height of the IPM image).

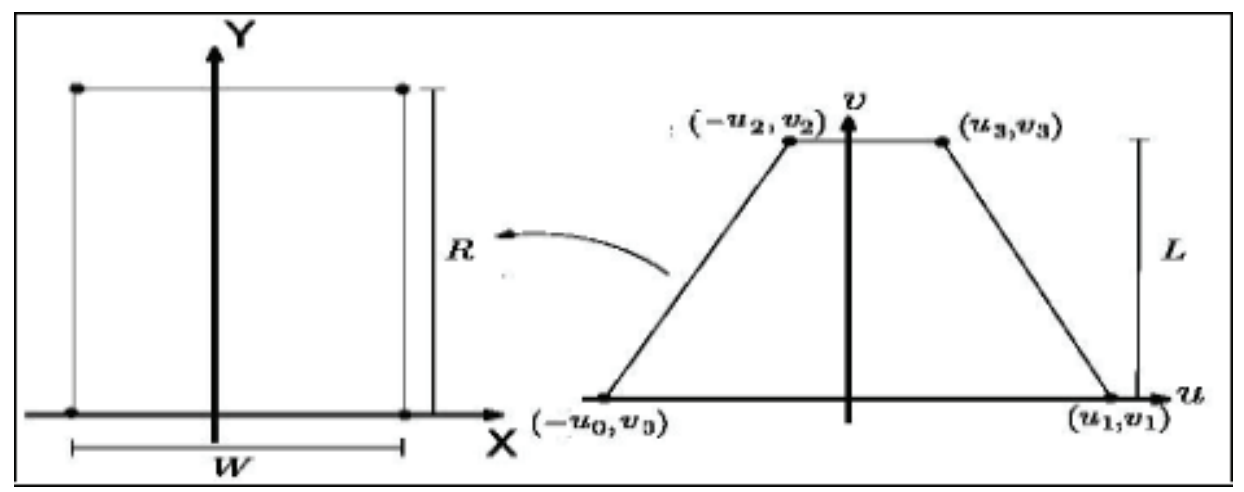

Fig. 6. Mapping of image coordinates $(u, v)$ into world coordinates $(X, Y)$. 
Figure 7 gives an example of the remapped top view image using the described inverse perspective mapping technique.

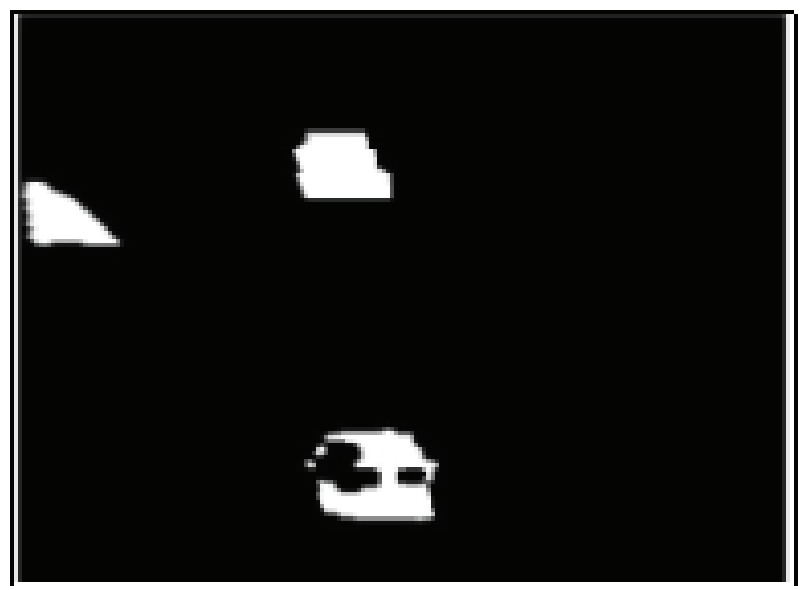

Fig. 7. The remapped IPM image for the image in Fig. 4.b.

\subsubsection{Building the Vehicles Tracking Diagram (VTD)}

The Vehicles Tracking Diagram is constructed in two steps as will be shown in the following subsections.

\subsubsection{Building the Intensity Distribution Graph (IDG)}

After binarization of the IPM image, a vertical intensity distribution graph (IDG) is built by summing all white pixels in each Y-level. To build the IDG for the IPM image of a certain frame F, we apply the following loop:

For each pin $\mathrm{Y}(\mathrm{Y}=0$ to $\mathrm{Y}=\mathrm{R})$ :

$$
\operatorname{IDG}(Y)=\sum_{X=0}^{X=W} \operatorname{IPM}(X, Y)
$$

Figure 8 shows an example of an IDG generated for a binary IPM image frame. As seen from Figure 8, the graph value at any Y-pin is proportional to both the number of vehicles $(\mathbf{N v})$ and the vehicle width in pixels (VWP). i.e.,

$$
I D G(Y)=\propto N v^{*} V W P
$$




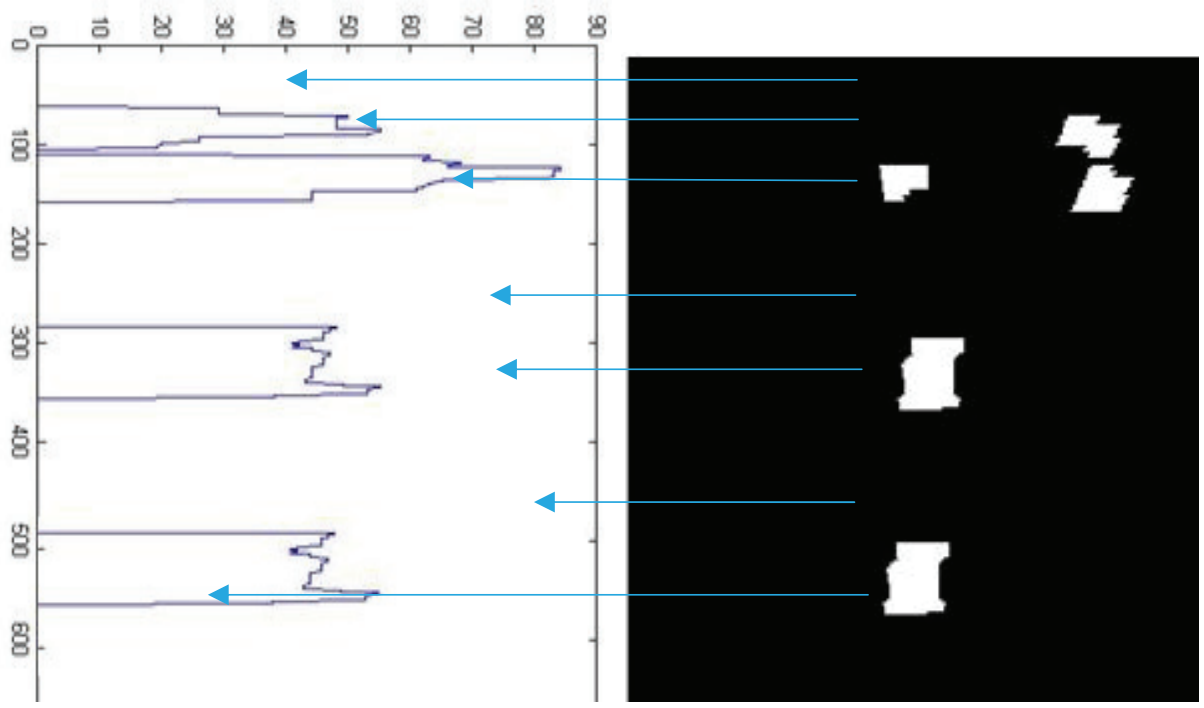

Fig. 8. The left graph is the Intensity Distribution Graph for the image in the right.

\subsubsection{Concatenating many Intensity Distribution Graphs to get one Vehicle Tracking Diagram}

In order to get a global picture of the traffic flow through the region of interest ROI within a limited period of time (Total Number of Frames TNF), we build what we called a Vehicles Tracking Diagrams (VTD) using the algorithm shown in Fig. 9.

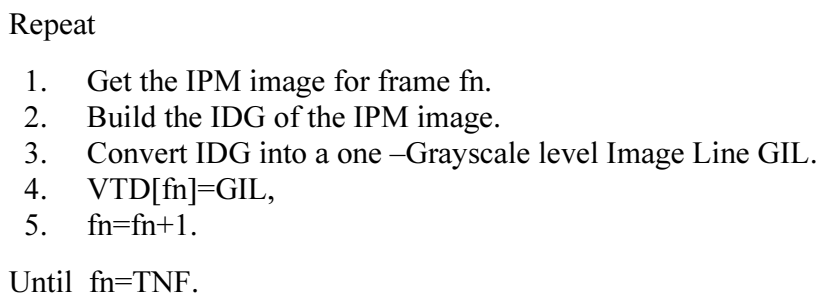

Fig. 9. The algorithm used in building the VTD.

Figure 10 illustrates the process of building up the VTD. After applying the previous algorithm we will get a final 2-D grayscale image as shown in Figure 11. 


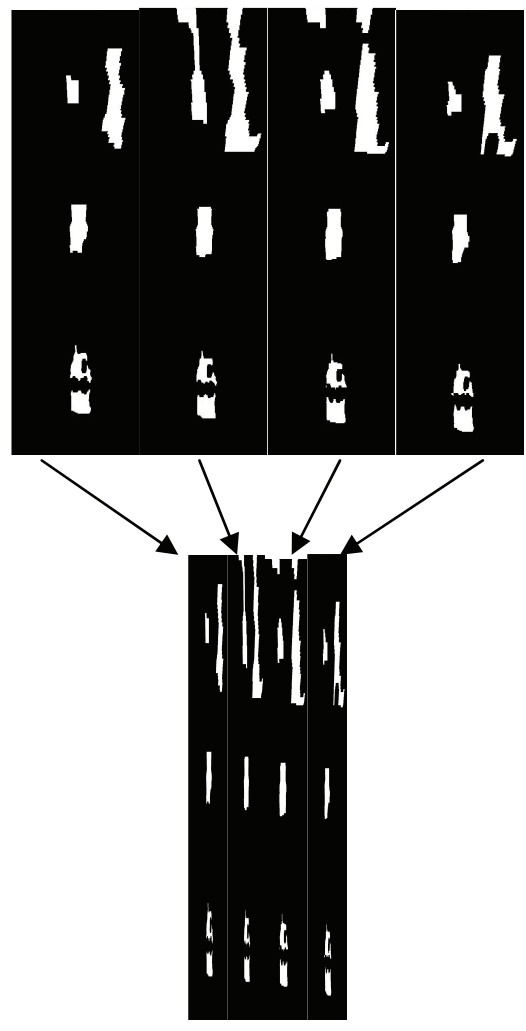

Fig. 10. The process of building the Vehicle Tracking Diagram.

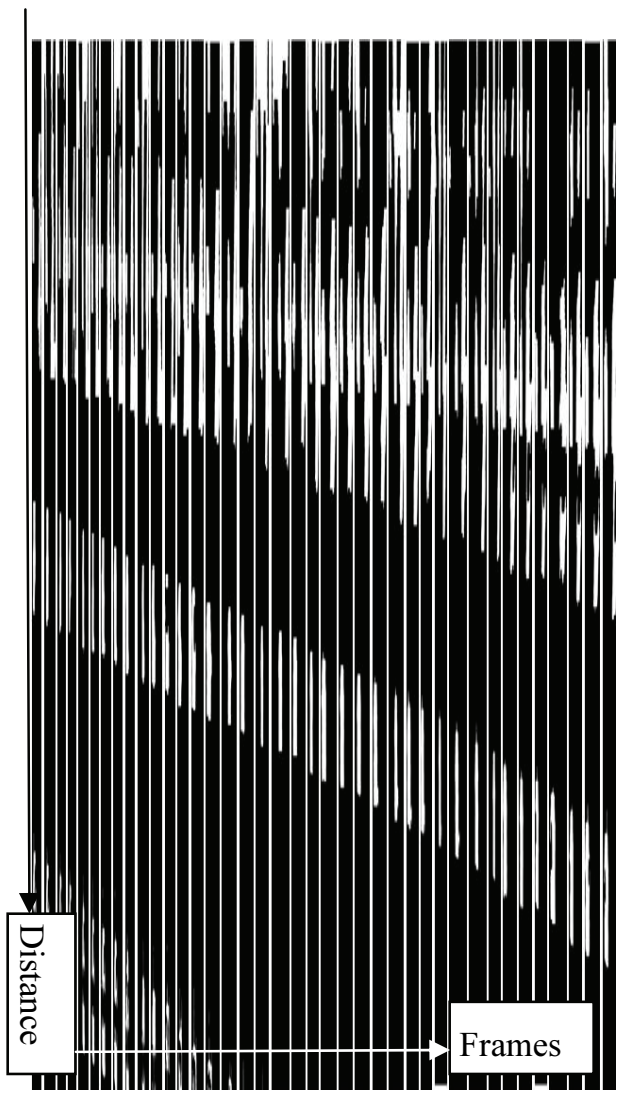

Fig. 11. The Vehicles Tracking Diagram (VTD).

The output $2 \mathrm{D}$ image is then rotated $90^{\circ}$ to get the rotated RVTD. The RVTD image will have a width equal to the inverse perspective image height (R) and a height equal to the Total Number of Frames (TNF) used per VTD. Figure 12 is a 3D representation of the VTD. The intensity at any point $(\mathrm{Y}, \mathrm{FN})$ represents the graph pin IDG(Y) at frame Fn, which is proportional to the number of vehicles at this Y-level and their widths. Hence, if we divide the intensity by the average vehicle width (AVWP) we can get the number of overlapped vehicles at this Ylevel. For example, in Figure 12, points 1, 2 and 3 refer to 3 different intensity levels indicating that the number of overlapped vehicles are 1,2 and 3 respectively. 


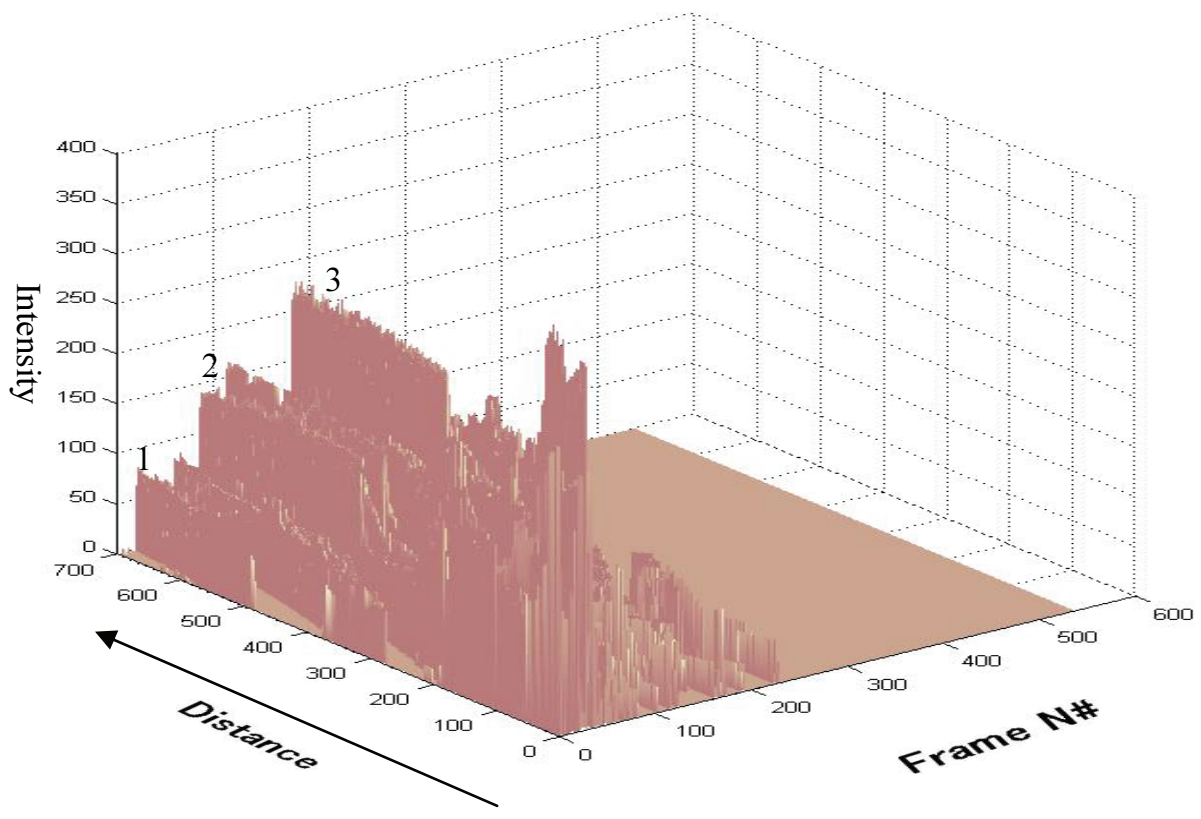

Fig. 12. 3D Representation of the Vehicles Tracking Diagram (VTD).

The average vehicle speed and average flow rate traffic parameters will be extracted from the RVTD as will be described in phase 3 .

\subsection{Phase 3: Traffic Parameters Extraction}

In this phase, the generated RVTD is processed using edge detection and Hough Line Transform (HLT) to detect the average vehicle speed and average flow rate. The steps needed are illustrated in the following subsections.

\subsubsection{Applying Hough Line Transform to get Vehicles Trajectory Lines.}

An actual 2D image of the Vehicles Tracking Diagram is shown in Figure 13. The 2D image shown in Fig. 13 is rotated $90^{\circ}$ to get the RVTD as shown in Fig. 14. 


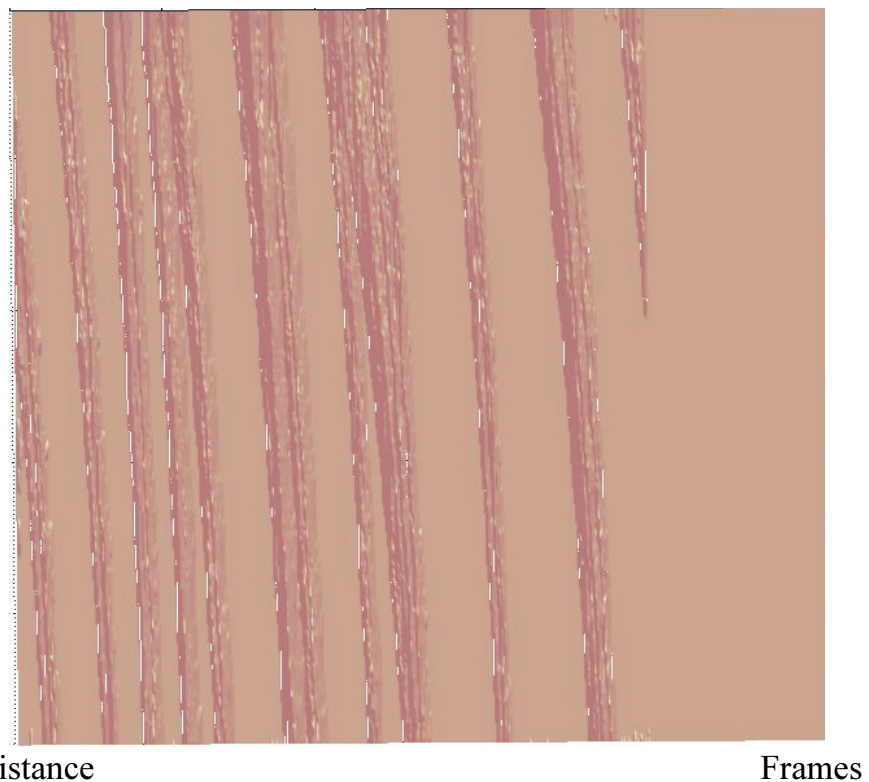

Fig. 13. 2D Vehicles Tracking Diagram (VTD).

Frames

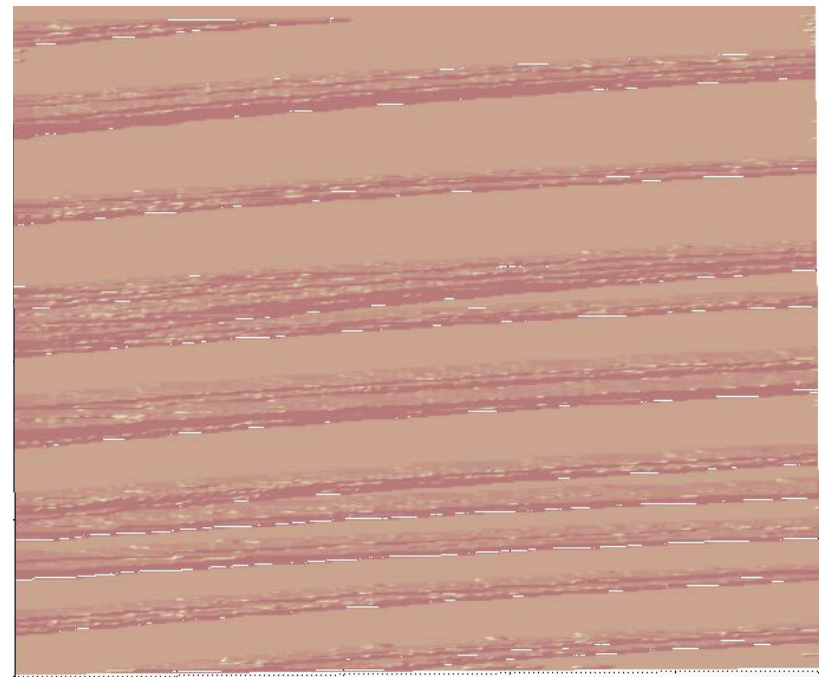

$\uparrow$

Distance

Fig. 14. RVTD image. 
To get the lines representing the vehicle trajectories, we apply edge detection on the RVTD image shown in Fig. 14 followed by HLT. This will result in many detected lines as shown in Fig. 15. Then we select only the lines near the camera (right end of the graph). Also vertical and horizontal lines are excluded, resulting in lines representing the beam boundaries (Bold lines) as shown in the same figure.

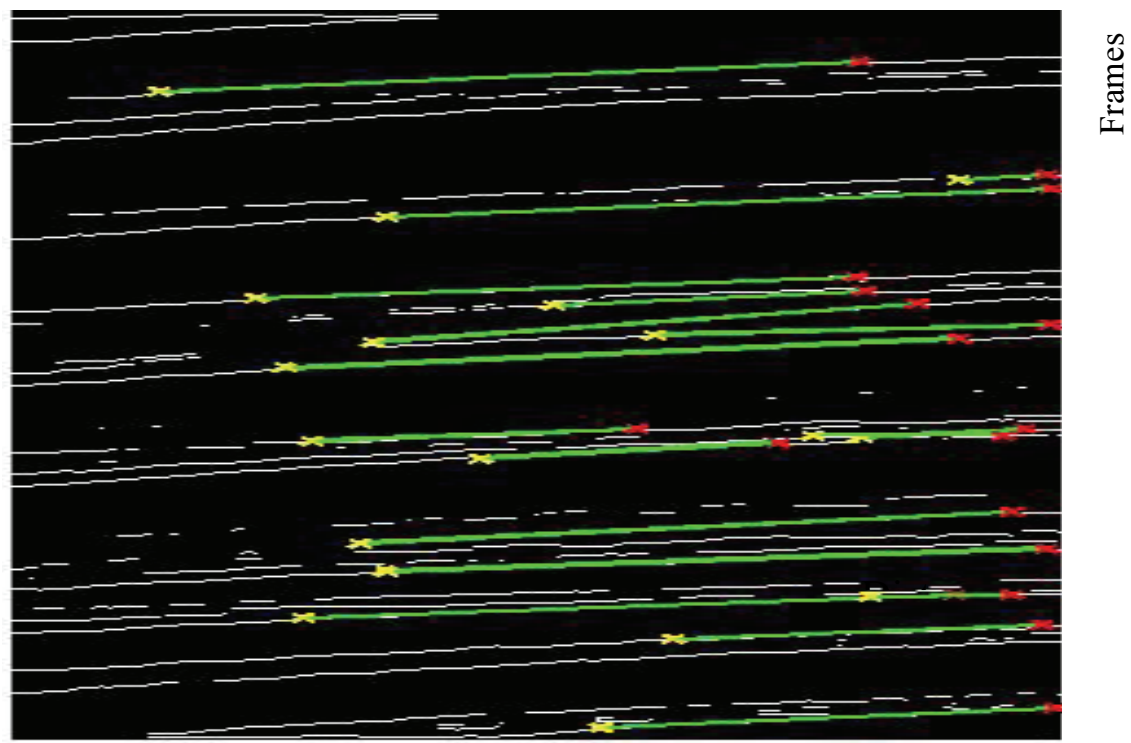

Distance

Fig. 15. RVTD after applying Edge detection and Hough Line Transform.

\subsubsection{Vehicle Average Speed Estimation.}

To estimate the vehicle average speed we first estimate the Average Slope AVSL $(\Delta t / \Delta y)$ of the selected lines (in the previous step). Since the average slope is the reciprocal of average vehicle speed in pixels per frame. Hence the Average Vehicle Speed in Kilometer per hour (AVSP) can be estimated as follows:

$$
A V S P=\frac{F s * 3600 * I M G S C L}{A V S L * 1000} \mathrm{Km} / \mathrm{h}
$$

Where $F s$ is the number of frames per second and IMGSCL is the image to real scale factor in meters per pixel which is deduced approximately as the actual vehicle length in meters (ACLM) divided by 
the average vehicle length in pixels $(A V L P)$. Hence equation 9 becomes as follows:

$$
A V S P=\frac{F_{S} * 3600 * A C L M}{A V S L * 1000 * A V L P} \mathrm{Km} / \mathrm{h}
$$

The average slope ( $A V S L)$ is used to deduce an important parameter which we called the beam width $(B W)$. The Beam Width $(B W)$ can be defined as the number of frames needed for the vehicle to leave the camera field of view.

From Fig. 16, $B W$ is estimated as the vehicle length in pixels multiplied by the average slope (AVSL) as follows:

$$
B W=A V L P * A V S L \text { pixels }
$$

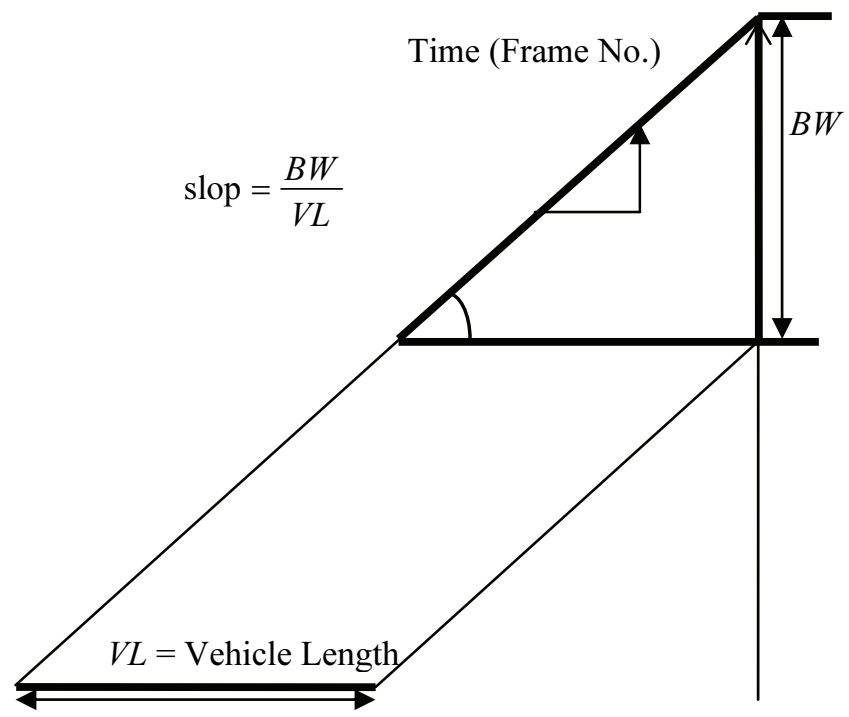

Fig. 16. The relation between the $B W$ and the Vehicle Length $(V L)$.

\subsubsection{Average Flow Rate Estimation}

The flow rate $(F R)$ can be measured in different units depending on the time interval needed to be monitored by the whole traffic system. In our system, we measure it in vehicles per minute. It can then be transformed to any units as needed by different systems. 
To measure the $F R$ we will count the vehicles leaving the camera field of view in the interval represented by the total number of frames $T N F$ that produced the RVTD.

To get the total number of vehicle (TNV) leaving the camera view, we sum the gray levels of the non black pixels vertically at the edge pin. Then this summation is divided by the beam width $(B W)$. As mentioned previously, in order to take overlapped vehicles into consideration the pixel gray values are proportional to the number of overlapped vehicles multiplied by their widths (8). Hence the actual number of vehicles will be as follows:

$$
\begin{gathered}
T N V(j)=\sum_{i=1}^{T N F} \frac{R V T D(i, j)}{B W^{*} A V W P} \\
\text { at } j=R
\end{gathered}
$$

To get the Flow Rate $(F R)$ which is defined previously as the total number of vehicles per minute we divide the total number of vehicles by the total number of frames TNF and then multiply the result by the number of frames per minute $\left(F s^{*} 60\right)$ as follows:

$$
F R=\left(\frac{T N V}{T N F}\right) * F_{S} * 60 \text { vehicles } / \mathrm{min}
$$

To get the average flow rate $A V F L$ we compute equation 13, 50 times starting from $\mathrm{j}=\mathrm{R}-50$ until $\mathrm{j}=\mathrm{R}$ and divide the result by 50 as defined in the following equation:

$$
A V F L=\frac{60 * F s}{T N F} * \frac{\left.\sum_{j=R-50}^{j=R} T N V(j)\right)}{50} \text { vhecles } / \mathrm{min}
$$

\section{Results}

The complete system has been designed and implemented using Matlab and the output results have been reported by experimenting the system with 3 video samples at different locations.

The video sequences were recorded using a Digital Camera with 4.0 Mega-Pixels, 10X Optical zoom and 40X Seamless Zoom. The height and width of each frame in the video were 480 and 640 pixels, respectively. The height of the Digital Camera above the road plane was about 7 meters. The frame rate used in the experiments was 30 frames 
per second. Table 1 shows the external camera parameters in each experiment as detected by the system.

Table 1. Camera External Parameters.

\begin{tabular}{|c|c|c|l|}
\hline Exp. N\# & Yaw angle $(\gamma)$ & Pitch angle $(\theta)$ & \multicolumn{1}{|c|}{ Remarks } \\
\hline 01 & $8.12^{\circ}$ & $36.66^{\circ}$ & Yaw- not satisfying the assumption. \\
\hline 02 & $1.35^{\circ}$ & $24.98^{\circ}$ & Yaw- nearly as assumed \\
\hline 03 & $0^{\circ}$ & $23.96^{\circ}$ & Yaw- as assumed \\
\hline
\end{tabular}

Figures 17.1 to 17.3 show the $3 \mathrm{D}$ representation of the vehicles tracking diagram (VTD) for the three experiments respectively. Figures 18.1 to 18.3 show the Vehicles Tracking Diagram (VTD) after applying edge detection and Hough line transform for the three experiments respectively. Table 2 lists the detected AVFR for the three experiments. The estimated flow rate was approximately equal to the actual flow rate except for experiment 1 . Table 3 lists the detected average vehicle speed AVSP for the 3 experiments. The estimated average vehicle speed was approximately equal to the actual average vehicle speed in experiments 2 and 3 , while experiment 1 gave a lower accuracy percentage because the $\gamma$-angle is not zero as assumed in our analysis.

Table 2. Detected Flow Rate.

\begin{tabular}{|c|c|c|c|c|c|c|}
\hline $\begin{array}{c}\text { Exp. } \\
\text { N\# }\end{array}$ & $\begin{array}{c}\text { Actual } \\
\text { NOV }\end{array}$ & $\begin{array}{c}\text { Actual Flow } \\
\text { Rate } \\
(\text { V/min })\end{array}$ & $\begin{array}{c}\text { Estimated } \\
\text { NOV }\end{array}$ & $\begin{array}{c}\text { Estimated Flow } \\
\text { Rate } \\
(\text { V/min })\end{array}$ & TNF & Accuracy\% \\
\hline 01 & 14 & 63 & 13.3 & 59.85 & 400 & 95.0 \\
\hline 02 & 16 & 72 & 15.5 & 69.75 & 400 & 96.8 \\
\hline 03 & 20 & 90 & 19.5 & 87.75 & 400 & 97.5 \\
\hline
\end{tabular}

Table 3. Measured average vehicle speed.

\begin{tabular}{|c|c|c|c|}
\hline Exp. N\# & Real AVSP $(\mathrm{km} / \mathrm{h})$ & Estimated AVSP $(\mathrm{km} / \mathrm{h})$ & accuracy\% \\
\hline 01 & 90 & 85.61 & 95.12 \\
\hline 02 & 80 & 76.95 & 96.18 \\
\hline 03 & 82 & 80.326 & 97.959 \\
\hline
\end{tabular}




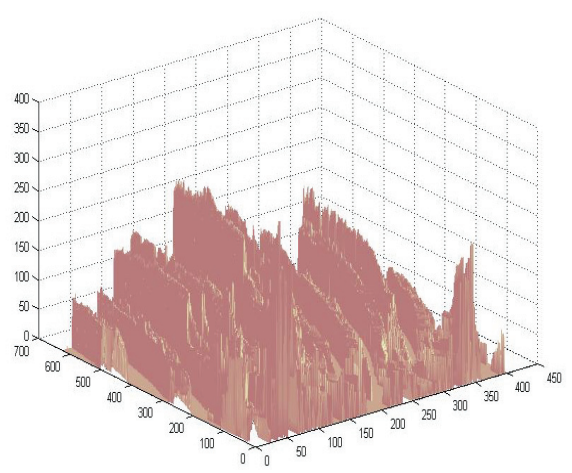

Fig. 17.1 3D Representation of the VTD in experiment 1.

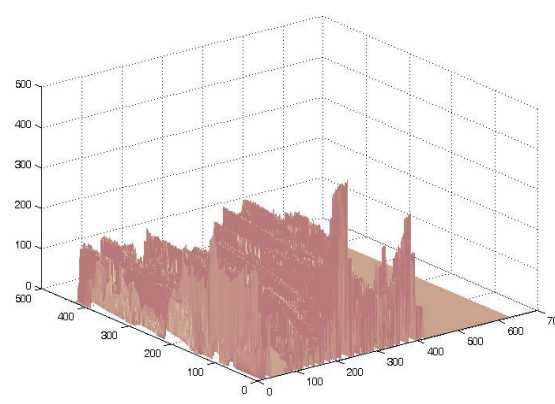

Fig. 17.2 3D Representation of the VTD in experiment 2.

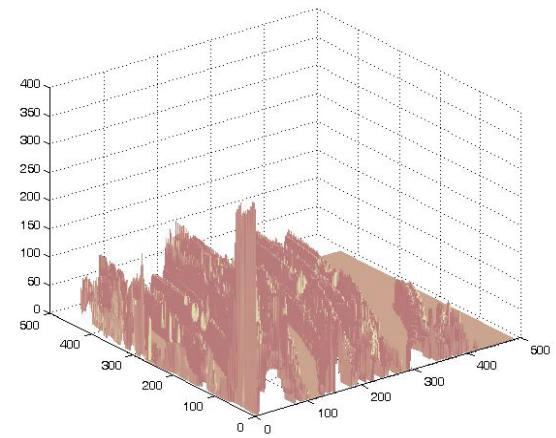

Fig. 17.3 3D Representation of the VTD in experiment 3.

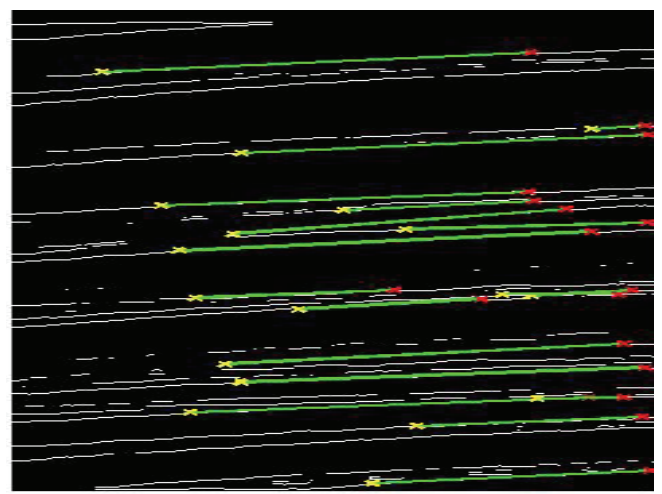

Fig. 18.1 VTD after applying Edge detection and Hough Line Transform in experiment 1.

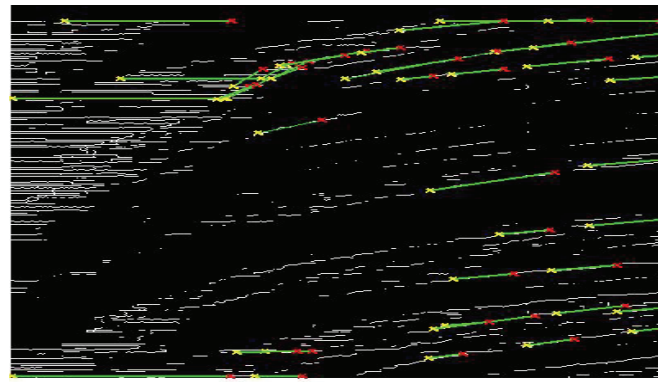

Fig. 18.2 VTD after applying Edge detection and Hough Line Transform in experiment 2.

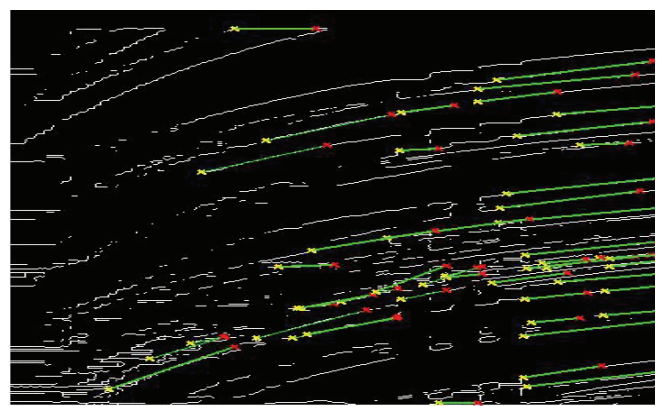

Fig. 18.3 VTD after applying Edge detection and Hough Line Transform in experiment 3. 
Comparison with other approaches:

In comparison to other approaches, we report the following points:

First: The system can count overlapped vehicles either horizontally or vertically since it does not depend on counting isolated vehicles but uses the area occupied by the vehicle as a basis for estimating the vehicle density. Hence if two vehicle images are connected, their contribution to the IDG will be double the value in case of single vehicle independently on their relative positions. This is in contrast to the entropy based solution $^{[11]}$ which recorded a large error rate in the case of overlapped vehicles.

Second: Vehicles changing lanes do not affect the estimation of average flow rate because any vehicle in any lane, its image will contribute to the corresponding pixels values of the resultant VTD. This in contrast to the case of window based tracking ${ }^{[3]}$ which suffers from missing the tracking of vehicles when they change lanes.

Third: Since our system uses Hough line transform to detect vehicle trajectory as piece-wise line segments, it will track any vehicle even if it disappears in some intermediate frames, leading to an acceptable accuracy when measuring the average speed. This is in contrast to the case of optical flow based tracking ${ }^{[8]}$ where tracking will fail or tracked object will be miss-oriented and if this is not detected the resultant speeds or vehicle counts will suffer from large error rates.

Fourth: The maximum speed that can be detected by our system is about $300 \mathrm{Km} / \mathrm{hr}$ or larger. The maximum speed can be measured based on the condition of overlapping between pictures of the same vehicle in any two consecutive frames. Assuming the vehicle length is about $4.2 \mathrm{~m}$ and the frame rate Fs is equal to $30 \mathrm{f} / \mathrm{s}$ then if the vehicle speed is 360 $\mathrm{km} / \mathrm{h}(100 \mathrm{~m} / \mathrm{s})$ then the vehicle travels $3.33 \mathrm{~m}$ per frame which means that the two images will overlap by $0.87 \mathrm{~m}$ (4.20-3.33). The same limitation is found in case of window based tracking ${ }^{[3]}$. In case of pixel based tracking like optical flow, to detect high speed ranges, a scale space approach is used to detect correspondences in the next frame which increases the computational time ${ }^{[8]}$.

Fifth: Automatic adaption of the system parameters is performed to ease the system installation in new locations without the need of predetermined points on real world coordinate. This is in contrast to most 
of the known approaches especially if the vehicle speed is to be estimated without IPM.

Sixth: Monitoring the traffic flow and vehicle speeds through the generated VTD is made easy for authorized persons to visualize the summary of the traffic state during a specified period graphically in one image in contrast to all other approaches where the recorded video file may be required to test every frame separately.

Seventh: Since IPM is performed as a major step in our system, measurement of vehicle speeds does not need further adjustment. This in contrast to other approaches which either need to do this major step or use predetermined points where the real distance between them should be known in advance.

Eighth: In our approach, vehicles having same model and color will not cause a problem in the calculated traffic parameters. This is in contrast to the feature based tracking techniques that should resort to other types of information to correct tracking of similar vehicles.

Finally, although our approach incorporates a dynamic background to compensate for variations of ambient lighting, it may suffer from the common problems associated with all the background subtraction based approaches such as shadows and cases where the vehicle color is nearly as the background color. Hence using very sophisticated techniques to model the background will enhance the overall behavior of our prototype system.

\section{Conclusion and Future Work.}

In this paper a new technique based on intensity distribution has been introduced to extract the traffic parameters: Average flow rate and average vehicle speed at the region of interest. The main contribution is the generation of the Vehicles Tracking Diagram which visually presents the traffic behavior within the ROI through a specified period of time in one picture. The intensity at any point of this diagram divided by the vehicle width gives the number of vehicles at this distance at a certain moment. The slope of the contour of the vehicle trajectory gives the vehicle speed. The summation of the pixel intensities at any Y-distance divided by the beam width and vehicle width gives the total number of vehicles leaving this point within the specified period of time. The 
experiments done on the video samples proved that the system can be used for measuring traffic parameters accurately in real applications.

Although the system speed is slow (about $5 \mathrm{~s}$ per frame), it can be enhanced using advanced image processing hardware or by using parallel processing techniques. The most important advantage of the system when compared to all other systems is that all its phases can be monitored graphically and visually which makes it more suitable for users to use and for researchers to enhance each phase or step individually. There are several issues that still need to be addressed concerning the adaptation of the system to work at night and also to test the system in different environmental conditions such as raining and fog.

\section{References}

[1] Jung, K.E.E. and Ho, Y.S., "Traffic Parameter Extraction using Video-based Vehicle Tracking”, Proc. IEEE Intelligent Transportation System Conf.'99, 1: 746-769, (1999).

[2] Zhencheng Hu, Chenhao Wang and Keiichi Uchimura," 3D Vehicle Extraction and tracking from Multiple Viewpoints for Traffic Monitoring by using Probability Fusion Map", Electronic Letters on Computer Vision and Image Analysis, 7(2): 110-119, (2008).

[3] Fathy, M. and Siyal, M. Y., "A Window-Based Image Processing Technique for Quantitative and Qualitative Analysis of Road Traffic Parameters", IEEE Transactions on Vehicular Technology, 47 (4), (1998).

[4] Kastrinaki, V., Zervakis, M. and Kalaitzakis, K., "A survey of video processing techniques for traffic applications", Image and Vision Computing, 21: 359-381, (2003).

[5] Beymer, D., McLauchlan, P., Coifman, B. and Malik, J., "A real-time computer vision system for measuring traffic parameters", IEEE Proc. of Conf. on Computer Vision and Pattern Recognition, Puerto Rico, 1: 496-501, (1997).

[6] Erhan INCE, "Measuring traffic flow and classifying vehicle types: A surveillance video based approach", Turk J Elec Eng \& Comp Sci., 19(4), CTUBITAK, doi: 10.3906/elk-0910266 (2011).

[7] Berthold, K.P. Horn and Brian, G. Rhunck, "Determining Optical Flow", Artificial Intelligence Laboratory, Massachusetts Institute of Technology, Cam bridge, MA 02139, U.S.A., (1998).

[8] Fatemeh Karimi Nejadasl a, Ben G.H. Gorte a, and Serge P. Hoogendoorn," Optical flow based vehicle tracking strengthened by statistical decisions", ISPRS Journal of Photogrammetry \& Remote Sensing, 61: 159-169, (2006).

[9] Viarani, E.,"Extraction of traffic information from images at DEIS", Proc. Int. Conf. on Image Analysis and Processing, pp: 1073-1076, (1999).

[10] Manchun Lei, Damien Lefloch, Pierre Gouton and Kadder Madani, "A video-based realtime vehicle counting system using adaptive background Method", IEEE International Conference on Signal Image Technology and Internet Based Systems (2008).

[11] W.-L. Hsu, H.-Y.M. Liao, B.-S. Jeng and K.-C. Fan, "Real-time traffic parameter extraction using entropy", IEE Proceedings of Vision, Image and Signal Process, doi: 10.1049/ip-vis:20040314 (2004). 
[12] Bas, E., "Road and Traffic Analysis from Video", M.S. Thesis, Electrical \& Computer Engineering, Koc University, July (2005).

[13] Thou-Ho Chen, Yu-Feng Lin, and Tsong-Yi Chen,” Intelligent Vehicle Counting Method Based on Blob Analysis in Traffic Surveillance", ICICIC '07: Proceedings of the Second International Conference on Innovative Computing, Information and Control, Sept., (2007).

[14] Marcos Nieto, Luis Salgado, Fernando Jaureguizar, and Juli'an Cabrera, "Stabilization of Inverse Perspective Mapping Images based on Robust Vanishing Point Estimation", Proceedings of the IEEE Intelligent Vehicles Symposium Istanbul, Turkey, June 13-15, (2007).

[15] Massimo Bertozzi, Albert Broggi, and Alessandra Fascioli, "Stereo inverse perspective mapping: theory and applications", Departimento di Ingegneria dell'Informazione, Universitir di Pana, Parma, Italy, (1997).

[16] Anderson A. G. A. Ribeiro, Leandro Lorenzett Dihl, and Cláudio R. Jung,"Automatic Camera Calibration for Driver Assistance Systems"; International conference on systems, signals and image processing; Budapeste, Hungria, (2006). 


\section{أسلوب جديد مبني على رسومات نوزيع الإضاءة لاستخلاص معدل التدفق المروري وسر عة العربة}

\section{جبرائيل الأمين محمد أبو سمرة، وعمر عبدالقادر}

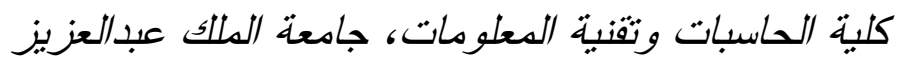

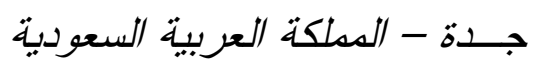

الستتخلص. يقدم هذا البحث نطامًا مرئيًا جديدًا يمكّن من تحليل

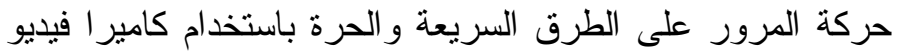

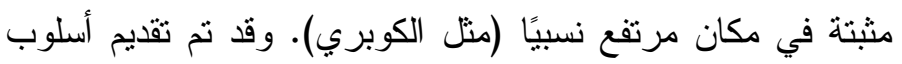

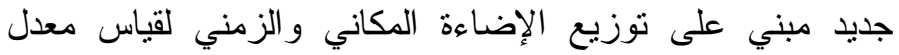

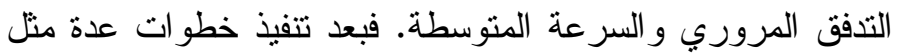

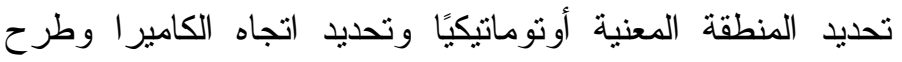

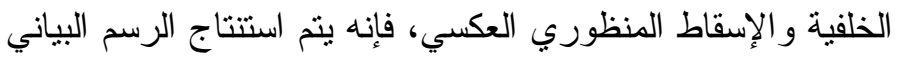

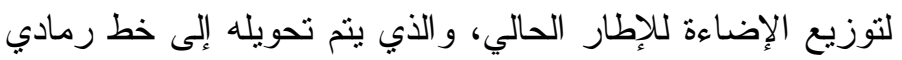

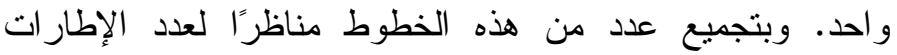

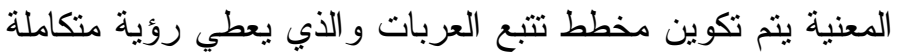

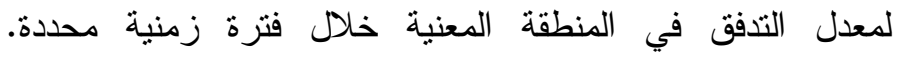

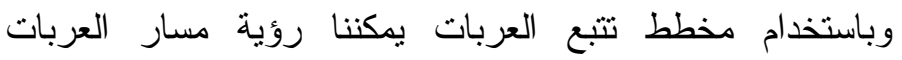

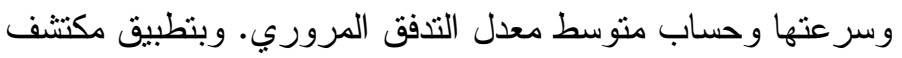
الحو اف ومحول هوف الخطي على مخطط تتبع العربات نتمكن من وند حساب متوسط سرعة العربات.

وقد تم بناء النظام باستخدام برنامج الماتلاب ونم تطبيقه تطبيقًا

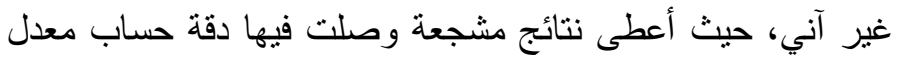

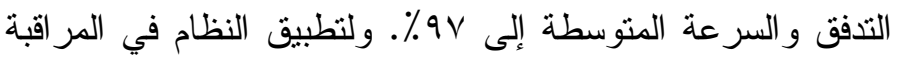
الآنية فإن النظام يتطلب المزيد من التحسين لزيادة سر عته لئه 